\title{
Análise Biofotométrica de Movimentos de Ombro e Cotovelo Relacionados com o Ganho Funcional e Tipos Cirúrgicos em Mulheres submetidas à Cirurgia Oncológica Mamária
}

doi: https://doi.org/10.32635/2176-9745.RBC.2020v66n2.895

\author{
Biophotometric Analysis of Shoulder and Elbow Movements Related to Functional Gains and Surgical Types in Women \\ Undergoing Breast Cancer Surgery \\ Análisis Bio-Fotométrico de los Movimientos del Hombro y Codo Relacionados con la Ganancia Funcional y los Tipos \\ Quirúrgicos en Mujeres Sometidas a Cirugía Oncológica de Mama
}

\author{
Lucas dos Santos Galaverna'; Maria Selma Duarte Nogueira²; Juliana Carolina Caixeta ${ }^{3}$; Frederico Tadeu Deloroso ${ }^{4}$; Eliane Maria \\ de Carvalho ${ }^{5}$
}

\section{Resumo}

Introdução: As cirurgias no câncer de mama ocasionam a redução da amplitude de movimento (ADM) de ombro, impactando na sua funcionalidade. A biofotometria é um recurso capaz de avaliar a ADM, mostrando precisão e reprodutibilidade. Objetivo: Mensurar a ADM de ombro e cotovelo e relacioná-las com o ganho funcional após intervençáo fisioterapêutica e o tipo cirúrgico em mulheres submetidas à cirurgia oncológica mamária. Método: Pesquisa observacional analítica, com 30 mulheres mastectomizadas em acompanhamento no Hospital do Câncer de Uberlândia, submetidas à avaliação biofotométrica, com marcaçóes em pontos padronizados nos membros superiores (MMSS) para análise de ADM em vista frontal e perfil, aplicação do questionário de disfunção de braço, ombro e mão (DASH) e questóes de atividades diárias propostas em uma ficha de anamnese antes e após quatro meses de intervenção. Resultados: Observou-se redução da $\mathrm{ADM}$ em todos os movimentos de ombro, as médias de abdução e flexão do ombro homolateral, antes e após quatro meses de intervenção fisioterapêtica, foram de 130,3 e 149,4 graus ( $\mathrm{p}=0,002)$; e 128,1 e 140 ( $\mathrm{p}=0,008)$, respectivamente. O escore do DASH diminuiu de $38,1$ para 32,15 ( $\mathrm{p}=0,047)$ e, nas questôes que envolvem abdução e flexão de ombro, houve maior aumento da porcentagem de respostas para "nenhuma dificuldade", com média de $23,08 \%$ para $42,86 \%$. Conclusão: A abdução e a flexão de ombro são os movimentos mais alterados no membro homolateral à cirurgia, entretanto, após quatro meses de fisioterapia, houve melhora da ADM acarretando ganho funcional, independentemente do tipo cirúrgico.

Palavras-chave: Mastectomia; Modalidades de Fisioterapia; Neoplasias da Mama; Amplitude de Movimento Articular.

\begin{abstract}
Introduction: Breast cancer surgeries cause a reduction in shoulder range of motion (SRM) impacting its functionality. Biophotometry is a resource that can assess SRM, showing precision and reproducibility. Objective: To measure the shoulder and elbow range of motion and relate them to the functional gains after physiotherapeutic intervention and surgical type in women undergoing breast cancer surgery. Method: An analytical observational research, with 30 mastectomized women being monitored at the Uberlândia Cancer Hospital, Brazil, submitted to biophotometric evaluation with graduation marks at standardized points in the upper limbs (ULs) in order to analyze the SRM in frontal and profile view, apply the Disabilities of the Arm, Shoulder and Hand (DASH) questionnaire and questions about the daily activities by filling in an anamnesis form before and after four months of intervention. Results: There was a reduction in SRM in all shoulder movements, the means of abduction and flexion scores of the homolateral shoulder, before and after four months of physiotherapeutic intervention, were 130.3 and 149.4 degrees $(\mathrm{p}=0.002)$ and 128.1 and $140(\mathrm{p}=0.008)$, respectively. The DASH score decreased from 38.1 to $32.15(\mathrm{p}=0.047)$ and, in issues involving shoulder abduction and flexion, there was greater increase in the percentage of responses answering "no difficulties", with mean from $23.08 \%$ to $42.86 \%$. Conclusion: Shoulder abduction and flexion are the most altered movements in the homolateral limb to the surgery, however, after four months of physiotherapy, there was an improvement in the SRM, leading to functional gain, regardless of the surgery type.

Key words: Mastectomy; Physical Therapy Modalities; Breast Neoplasm; Range of Motion, Articular.
\end{abstract}

Resumen

Introducción: Las cirugías de cáncer de mama causan una reducción de la amplitud de movimiento del hombro (AMH), que afecta su funcionalidad. La bio-fotometría es un recurso capaz de evaluar AMH, mostrando precisión y reproducibilidad. Objetivo: Averiguar la amplitud de los movimientos del hombro y codo y relacionarlos con la ganancia funcional después de la fisioterapia y la intervención quirúrgica en mujeres sometidas a cirugía de cáncer de mama. Método: Investigación analítica observacional con 30 mujeres mastectomizadas, en acompañamiento en el Hospital del Cáncer de la ciudad de Uberlândia, Brasil, sometidas a evaluación bio-fotométrica con marcaciones en puntos estandarizados en los miembros superiores (MMSS) para análisis de la AMH en vista frontal y perfil, aplicación del cuestionario de disfunción de brazo, hombro y mano (DASH) y preguntas sobre las actividades diarias propuestas en formato de anamnesis, antes y después de cuatro meses de intervención. Resultados: Hubo una reducción en la AMH en todos los movimientos de los MMSS, la abducción y flexión media del hombro homolateral, antes y después de cuatro meses de la intervención fisioterápica, fue 130,3 y 149,4 grados ( $\mathrm{p}=0,002)$; y 128,1 y 140 ( $\mathrm{p}=0,008)$, respectivamente. La puntuación mediana del DASH disminuyó de 38,1 a $32,15(\mathrm{p}=0,047)$, y en las preguntas relacionadas con la abducción y la flexión del hombro, hubo un mayor aumento en el porcentaje de respuestas para "sin dificultad", con un promedio de 23,08\% a 42,86\%. Conclusión: La abducción y flexión del hombro son los movimientos más alterados en el miembro homolateral a la cirugía, entretanto, después de cuatro meses de fisioterapia, hubo una mejora en la $\mathrm{AMH}$, lo que llevó a una mejora en la capacidad funcional, independientemente del tipo de cirugía.

Palabras clave: Mastectomía; Modalidades de Fisioterapia; Neoplasias de la Mama; Rango del Movimiento Articular.

\footnotetext{
${ }^{1}$ Faculdade de Educação Física e Fisioterapia. Universidade Federal de Uberlândia (UFU). Uberlândia (MG), Brasil. Orcid iD: https://orcid.org/0000-0001-5581-7876

${ }^{2}$ Faculdade de Educação Física e Fisioterapia. UFU. Uberlândia (MG), Brasil. Orcid iD: https://orcid.org/0000-0002-3236-0815

${ }^{3}$ Faculdade de Educação Física e Fisioterapia. UFU. Uberlândia (MG), Brasil. Orcid iD: https://orcid.org/0000-0002-0731-3224

${ }^{4}$ Faculdade de Educação Física e Fisioterapia. UFU. Uberlândia (MG), Brasil. Orcid iD: https://orcid.org/0000-0001-8714-2156

${ }^{5}$ Faculdade de Educação Física e Fisioterapia. UFU. Uberlândia (MG), Brasil. Orcid iD: https://orcid.org/0000-0002-3977-9117

Endereço para correspondência: Lucas dos Santos Galaverna. Rua Pedro Silotto, 108 - Centro. Serra Negra (SP), Brasil. CEP 13930-000. E-mail: lucas_galaverna98@hotmail.com
} 


\section{INTRODUÇÃO}

O câncer de mama é uma das principais doenças crônicas náo transmissíveis, englobando mais de 100 tipos de desordens de crescimento e multiplicação celular que invadem tecidos e órgãos, de maneira que o sistema de defesa do organismo é incapaz de deter ${ }^{1}$. No Brasil, de acordo com o Instituto Nacional de Câncer José Alencar Gomes da Silva (INCA), a estimativa, para cada ano do triênio 2020-2022, é de 625 mil novos casos de câncer, sendo 66.280 casos para o câncer de mama, o qual corresponde um risco estimado de 61,61 novos casos a cada 100 mil mulheres ${ }^{1}$. O prognóstico é variável para cada caso e depende de fatores como presença de metástases, comprometimentos de linfonodos e extensão da doença ${ }^{2}$. O câncer de mama apresenta alta mortalidade em mulheres, e as estratégias para seu controle possuem dependência de políticas públicas de saúde e agilidade para o tratamento rápido e eficiente, visto que a identificação e intervenção precoce reduzem a morbimortalidade da doença ${ }^{3}$.

As repercussóes do tratamento oncológico da mama são negativas em muitos aspectos na vida da mulher, como psicológica, social e física, uma vez que, após a cirurgia mamária, a imagem corporal é alterada ${ }^{4}$. Além disso, a capacidade funcional de membro superior (MS) é comprometida, em virtude dos diferentes procedimentos cirúrgicos e imobilização pós-cirúrgica, ademais, nas cirurgias radicais, os músculos peitoral maior e peitoral menor são retirados, acarretando redução da força e movimento do MS homolateral ao procedimento5.

As alteraçôes físicas decorrentes do tratamento cirúrgico são encontradas em cerca de $63,5 \%$ das mulheres operadas e, entre as alteraçôes, a amplitude de movimento articular (ADM) ativa de MS é a mais frequente, principalmente nos movimentos de flexão e abdução de ombro ${ }^{6,7}$. Para reduzir tais complicaçóes, a fisioterapia engloba técnicas como a cinesioterapia e eletroterapia. Uma revisão realizada em $2020^{8}$ demonstrou que as intervençóes compostas por fortalecimento global, alongamentos, mobilização articular e terapia vibratórias apresentam grandes benefícios na recuperaçáo funcional em mulheres pós cirurgia oncológica mamária.

Existem diversos modos de mensurar a $\mathrm{ADM}^{9}$. A biofotometria é recurso que pode ser utilizado para tal, seja ela passiva ou ativa ${ }^{10}$. Esse instrumento tem sido utilizado em diversas áreas para elaboração de diagnósticos físicos-funcionais, já que é acessível na prática clínica com grande fidedignidade, baixo custo, facilidade de manuseio, alta precisão e reprodutibilidade, além de reduzir o erro entre avaliadores e intra-avaliadores, diferente de outros tipos de mensuração ${ }^{10-12}$.
Embora a literatura apresente grande quantidade de estudos acerca do impacto funcional no tratamento oncológico mamário, poucas pesquisas demonstram os impactos que os diferentes tipos cirúrgicos influem sobre a $\mathrm{ADM}$, e o quanto o ganho da $\mathrm{ADM}$ após uma intervenção fisioterapêutica de rotina ambulatorial pós-cirúrgica altera a funcionalidade de membros superiores (MMSS). Majoritariamente, os estudos apresentam dados sobre um único momento da paciente com variáveis individuais, sem considerar o tempo decorrido entre a cirurgia e a chegada ao tratamento fisioterapêutico ${ }^{13-16}$.

Diante do exposto, este trabalho se propôs a analisar a ADM dos ombros e cotovelos de mulheres submetidas a cirurgias oncológicas mamárias e relacioná-las com o ganho funcional ocasionado pelo tratamento fisioterapêutico individualizado por quatro meses, identificando o possível impacto que os diferentes tipos cirúrgicos, radical ou conservador, ocasionam na $\mathrm{ADM}$ dessas articulações.

\section{MÉTODO}

Estudo observacional analítico correlacional, aprovado pelo Comitê de Ética em Pesquisa com Seres Humanos da Universidade Federal de Uberlândia sob o número 2.731.732.

A amostragem foi não probabilística, do tipo conveniência, por se tratar de um estudo exploratório que visou a identificar a relação entre a amplitude articular, mensurada pela biofotometria, e seu impacto na funcionalidade de MMSS mensurada pelo questionário de disfunçáo do ombro, braço e máo (do inglês, Disabilities of the Arm, Shoulder and Hand - DASH), em sua versão completa, validado e traduzido para o português em um estudo com 65 pacientes com artrite reumatoide, o qual demonstra alto nível de reprodutibilidade e um intervalo de confiança de $90 \%{ }^{17,18}$.

As coletas foram realizadas no ambulatório de Fisioterapia do Hospital de Clínicas/Hospital do Câncer de Uberlândia, no período de maio de 2018 a junho de 2019. As voluntárias foram convidadas a participar da pesquisa $\mathrm{e}$ orientadas quanto às informaçóes sobre as coletas. Após isso, as que concordaram assinaram o Termo de Consentimento Livre e Esclarecido, iniciando assim a pesquisa.

Foram incluídas mulheres submetidas à cirurgia oncológica de mama radical e conservadora, com ou sem esvaziamento axilar, e liberadas pelo médico para a execução de atividade física supervisionada; os critérios de exclusão foram a presença de ferimentos e/ou lesōes na regiáo do peito, metástase e fraturas patológicas de MS.

Os dados sociodemográficos, de saúde e tratamento foram colhidos no prontuário (idade, tempo entre a cirurgia 
e a avaliação, cirurgia no lado dominante ou não, cirurgia conservadora ou radical) e tratamentos coadjuvantes (quimioterapia, radioterapia, hormonioterapia e combinaçóes entre estas). Em seguida, foram aplicados o questionário DASH, o questionário de funcionalidade em atividades específicas propostas na ficha de avaliação e anamnese como uma análise complementar da capacidade funcional, e a coleta da biofotometria.

As coletas dessas informaçóes foram no dia do início do tratamento fisioterapêutico, e as variáveis dependentes foram coletadas na reavaliação realizada quatro meses após o tratamento individualizado e personalizado para cada paciente, objetivando o ganho funcional, sem modificaçôes ao longo deste estudo, composto por eletroterapia e cinesioterapia, realizado duas vezes por semana, por 50 minutos em fase ambulatorial, por fisioterapeutas especialistas em oncologia, sendo o tempo entre as avaliaçôes considerado a média para a recuperação de $\mathrm{ADM}^{19}$. Os recursos utilizados foram correntes excitomotoras (FES e corrente russa) por 20 a 30 minutos com intensidade até 120 miliamperes de acordo com a sensibilidade da paciente, com eletrodos posicionados em regiấo escapular, extensão dos MMSS e peitoral, alongamentos passivos dos músculos peitoral e trapézios e fortalecimento global de MMSS com auxílio de faixas elásticas e halteres de até $3 \mathrm{Kg}$ com intensidade moderada determinada pela percepçáo subjetiva de esforço da paciente.

\section{AVALIAÇÃO DA AMPLITUDE DE MOVIMENTO DE OMBROS PELA BIOFOTOMETRIA}

A biofotometria é um método rápido, de fácil manuseio, alta reprodutibilidade e pode ser utilizada para mensuração de angulaçóes em MS e diagnósticos funcionais. Esta já foi utilizada por diversos autores em diferentes áreas, mostrando grande utilidade $\mathrm{e}$ fidedignidade em seus resultados, possibilitando, também, o arquivamento de registros. Além disso, apresenta boa difusão e aceitação pelos profissionais que analisam o movimento humano em virtude do seu alto nível de objetividade e concordância entre avaliadores em relação a outras técnicas de mensuração de $\mathrm{ADM}$, como a goniometria, motivos pelos quais se utilizou tal método $^{11,20,21}$.

Para a realização da biofotometria, foi utilizada uma câmera da marca Canon modelo EOS Rebel T6 EF-S 18-55 f/3.5-5.6 III com boa resolução para o registro das imagens. A câmera foi posicionada paralelamente ao solo em um tripé fixo e nivelado. A distância e a altura do tripé foram padronizadas em 1,50 metro. $\mathrm{O}$ ambiente para a realização das análises apresentava boa iluminação, com o mínimo de interferências possíveis ${ }^{22}$.
As voluntárias foram analisadas na posição sentada para minimizar movimentos compensatórios do corpo observados na posição ortostática. A cadeira utilizada foi padronizada para todas as voluntárias, a qual não impedia a execuçáo dos movimentos analisados e fornecia ajustes para adaptação antropométrica (altura do assento), caso necessário, para que os pés da voluntária ficassem em total apoio no solo. As mulheres receberam instruçóes verbais para o posicionamento e vestimenta em todas as fotos. Os pontos anatômicos foram demarcados com cilindros de alto relevo com $150 \mathrm{~mm}$ de diâmetro na cor branca. Os pontos determinados foram extremidade acromial da clavícula direita e esquerda; cabeça do rádio direito e esquerdo, processo estiloide dos rádios direito e esquerdo, e centro dos punhos direito e esquerdo. As fotos foram realizadas no plano frontal, em vista anterior, e plano sagital, com vistas laterais ${ }^{23}$. No plano frontal, foi analisado o movimento de abdução de ombro. No plano sagital, foram analisados os movimentos de flexão e extensão de ombros e a flexão e extensão de cotovelos. Ambos os membros, homolateral e contralateral à cirurgia, foram avaliados, para que houvesse determinaçáo do grau de acometimento do membro homolateral à cirurgia, sendo o contralateral tomado como referência normal para as voluntárias.

Para análise quantitativa da biofotometria, foi utilizado o software Kinovea, no qual os pontos pré-demarcados foram utilizados como referência para medição da angulação, de alta facilidade no manuseio.

Para análise dos movimentos de abdução, flexão e extensão de ombro, o eixo do ângulo foi fixado do acrômio, uma das retas perpendicular ao solo e outra reta passam pela cabeça do rádio (Figuras 1-a, 1-b e 1-c). E, para análise de flexão de cotovelo, o eixo foi marcado na cabeça do rádio, uma das retas passa pela projeção do acrômio e outra reta segue até o processo estiloide do rádio (Figura 1-d).

\section{AVALIAÇÃO DA CAPACIDADE FUNCIONAL DE MEMBROS SUPERIORES}

Para avaliar a capacidade funcional de MS, foi utilizado o questionário DASH.

O DASH consta de 30 questôes autoaplicáveis, com itens que informam sobre o grau de dificuldade no desempenho de atividades; a intensidade dos sintomas de dor, fraqueza, rigidez e parestesia; o comprometimento de atividades sociais; a dificuldade para dormir e o comprometimento psicológico, tendo como referência a semana anterior à aplicação do instrumento.

As questóes do instrumento informam sobre o estado de saúde da mulher na última semana, sendo 21 questôes sobre o nível de dificuldades para desempenhar atividades 

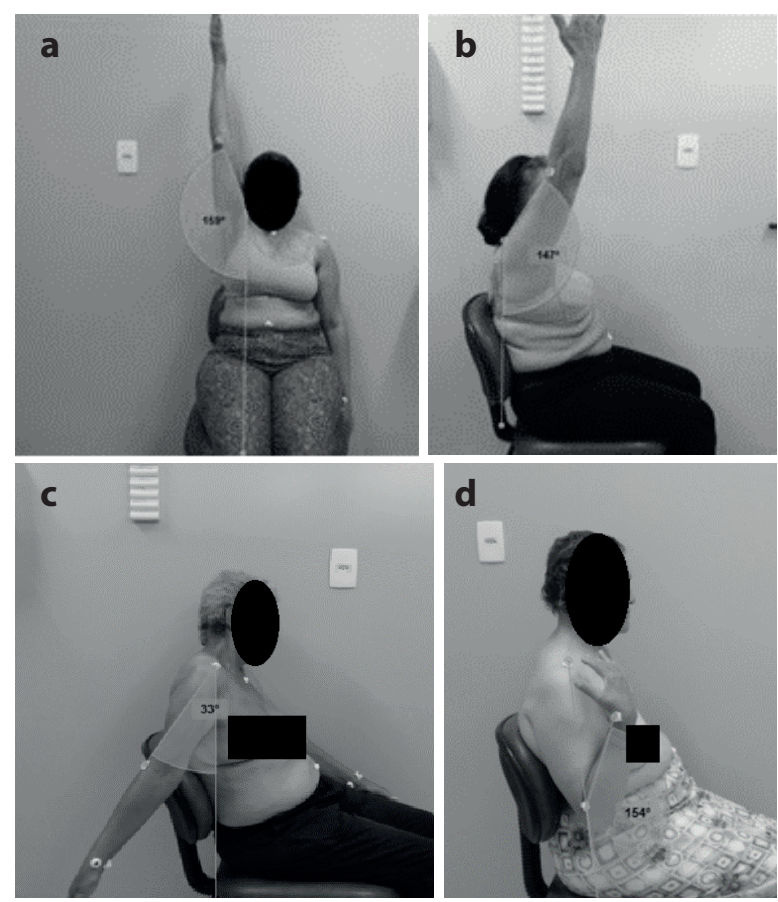

Figura 1. Análise biofotometria pelo Kinovea sobre abdução (1-a); flexão (1-b); extensão de ombro (1-c); flexão de cotovelo (1-d)

físicas por causa do acometimento no braço, ombro ou mão; cinco questôes referentes à gravidade dos sintomas como dores, parestesia, fraqueza e rigidez; e quatro questôes relativas ao impacto da condição patológica nas atividades sociais, de trabalho, sono e autoimagem. Cada questão do DASH possui cinco opçóes de respostas, variando de um, para nenhuma dificuldade ou sintoma, até cinco, para incapacidade para desempenhar a tarefa ou extrema gravidade de sintoma. Esses valores foram transformados em um escore de 100, subtraindo $1 \mathrm{e}$ multiplicando por 25. Essa transformação foi feita para comparar os escores com outras escalas de 0 a 100 . O escore alto indica grande disfunção, a partir da equação: [(Soma das respostas / 30) -1] X $25^{24}$.

No questionário DASH, quanto maior o escore, maior a incapacidade; ou seja, pior será a capacidade funcional. Isto se deve ao fato de o DASH ter sido criado na perspectiva $\mathrm{da}$ incapacidade; ou seja, maiores escores brutos indicam maior comprometimento da capacidade funcional ${ }^{24}$.

Além do DASH, a funcionalidade também foi analisada a partir do protocolo de avaliação elaborado no próprio ambulatório de Fisioterapia como um material suplementar para avaliação da capacidade funcional, composto por oito atividades em que a paciente deveria classificar de 1 a 4, sendo 1 - Não consegue fazer sozinha; 2 - Realiza com muita dificuldade; 3 - Realiza com pouca dificuldade; 4 - Sem dificuldade para realizar. Ao final da pesquisa, serão vistas as porcentagens de respostas. Quanto mais alta a porcentagem de participantes que responderam 1 ou 2, pior a funcionalidade.

\section{ANÁLISE ESTATÍSTICA}

As variáveis analisadas foram armazenadas em um banco de dados no Microsoft Excel para posterior análise no programa "RStudio" para obtenção das análises descritivas e comparativas com todos os direitos autorais do programa reservados à empresa originária. Para a análise descritiva, foram calculadas medidas de tendência central (média e mediana) e de dispersão (valor mínimo e máximo e desvio-padrão) para as variáveis contínuas. As medidas de frequências relativas e absolutas foram calculadas para as variáveis categorizadas, para melhor compreensão dos dados.

$\mathrm{Na}$ análise inferencial, foi utilizado o teste de normalidade de Shapiro-Wilk para as variáveis dependentes e, após confirmação de distribuição normal, utilizou-se o teste t pareado entre a avaliação e reavaliação e correlação bisserial quando se tratava de uma das variáveis nominais, bem como o teste de hipóteses com intervalo de confiança de $95 \%$; portanto, foram aceitos valores com $\mathrm{p}<0,05$ para dados estatisticamente significantes.

Os valores obtidos pelas correlaçóes ( $r$ ) foram interpretados de acordo com os limites estabelecidos por Weber e Lamb ${ }^{25}$ : 0,00-0,19=nenhuma ou ligeira; 0,20-0,39=leve; $0,40-0,69=$ moderada; $0,70-0,89=$ alta; e $0,90-1,00=$ muito alta ${ }^{25}$.

\section{RESULTADOS}

Foram analisadas 30 voluntárias na primeira avaliação e 19 na reavaliação. As causas da redução amostral se contiveram a óbito (1); evolução para cuidados paliativos (2); e desistência para a reavaliação (8). Para as análises descritivas, foram utilizados dados das voluntárias que foram avaliadas antes de iniciar o tratamento fisioterapêutico e, para as análises comparativas, apenas as voluntárias que foram reavaliadas após quatro meses.

A média de idade das voluntárias foi de $54,7 \pm 9,2$ anos, e os tipos cirúrgicos contemplaram cirurgias radicais (23) e conservadoras (7), sendo que 17 mulheres realizaram a cirurgia no lado superior dominante e 13 no lado contralateral. A média de tempo entre a cirurgia e a avaliaçáo foi de $10,43 \pm 15,61$ meses. Realizavam tratamento coadjuvante 28 voluntárias, sendo eles: quimioterapia (2), radioterapia (4), hormonioterapia (2), associando quimioterapia e radioterapia (11) e associando os três tipos (9).

$\mathrm{Na}$ Tabela 1, estấo representados os valores médios e desvios-padrão das angulações de movimentos de ombro e cotovelo medidos pela biofotometria. Pode-se observar que os movimentos de abdução e flexão de ombro dos membros contralaterais à cirurgia são maiores em relação aos movimentos do membro homolateral, bem como o aumento das angulaçôes na segunda avaliação. 
Tabela 1. Valores das angulações de movimentos de ombros e cotovelo segundo biofotometria

\begin{tabular}{lccc}
\multicolumn{1}{c}{ Movimento\Fase da pesquisa } & Avaliação & Reavaliação & p=valor \\
\hline Abdução de ombro homolateral & $130,4 \pm 29,7$ & $149 \pm 18,7$ & 0,0021 \\
Abdução de ombro contralateral & $139,5 \pm 33,8$ & $159,8 \pm 9,7$ & 0,0671 \\
p=valor & 0,0008 & 0,032 & 0,008 \\
Flexão de ombro homolateral & $129,7 \pm 36,1$ & $143 \pm 19,6$ & 0,086 \\
Flexão de ombro contralateral & $143,9 \pm 30,2$ & $156,4 \pm 14,4$ & 0,044 \\
P=valor & 0,006 & 0,067 & 0,013 \\
Extensão de ombro homolateral & $43,9 \pm 18,2$ & $48 \pm 17,2$ & $49,6 \pm 16,3$ \\
Extensão de ombro contralateral & $42,2 \pm 14,2$ & 0,098 & 0,865 \\
P=valor & 0,225 & $153,3 \pm 6$ & 0,734 \\
Flexão de cotovelo homolateral & $154,6 \pm 8,4$ & $157 \pm 6,2$ & 0,969 \\
Flexão de cotovelo contralateral & $157,6 \pm 6,5$ & 0,159 & 0 \\
P=valor & & & 0 \\
\hline
\end{tabular}

Nota: Nas linhas: comparação entre avaliação e reavaliação; nas colunas: comparaçáo entre lado homolateral e contralateral; p<0,05

Ao separar os dados de acordo com os tipos cirúrgicos (radical e conservador), nota-se que há diferença dos ganhos de angulação, principalmente dos movimentos de abdução de ombro, que, na cirurgia conservadora, foram de $136,3 \pm 24,2$ para $151,5 \pm 19,1(\mathrm{p}=0,011)$ no lado homolateral e $140,3 \pm 33,14$ para $162,1 \pm 8,3(\mathrm{p}=0,224)$ no lado contralateral; e flexão de ombro, a qual foi de $138,6 \pm 28,5$ para $142,7 \pm 21,2(\mathrm{p}=0,036)$ no lado homolateral e $146,3 \pm 29$ para $156,7 \pm 8,6(\mathrm{p}=0,561)$ no lado contralateral. Já na cirurgia radical, o movimento de abduçáo de ombro foi em média de $108,2 \pm 40,72$ para $138 \pm 14,1$ ( $\mathrm{p}=0,016)$ no lado homolateral e 136,6 ${ }_{ \pm} 40,2$ para $146 \pm 4,25(\mathrm{p}=0,351)$ no lado contralateral; e a flexão de ombro foi de $95,6 \pm 45,10$ para $145 \pm 8,48(\mathrm{p}=0,017)$ no lado homolateral e de $134,6 \pm 36,5$ para $154,5 \pm 43,1$ $(\mathrm{p}=0,234)$ no lado contralateral.

Com relação às questóes de atividades propostas pela avaliação funcional, observa-se que as porcentagens de respostas que mostram melhores funcionalidades de MS aumentaram; ou seja, a porcentagem de voluntárias que responderam “4” aumentou após quatro meses de intervenção; sendo 1 - Não consegue fazer sozinha; 2 Realiza com muita dificuldade; 3 - Realiza com pouca dificuldade; 4 - Sem dificuldade para realizar (Figura 2).

O escore desse questionário foi calculado somando-se os valores atribuídos para cada atividade, assim sendo,

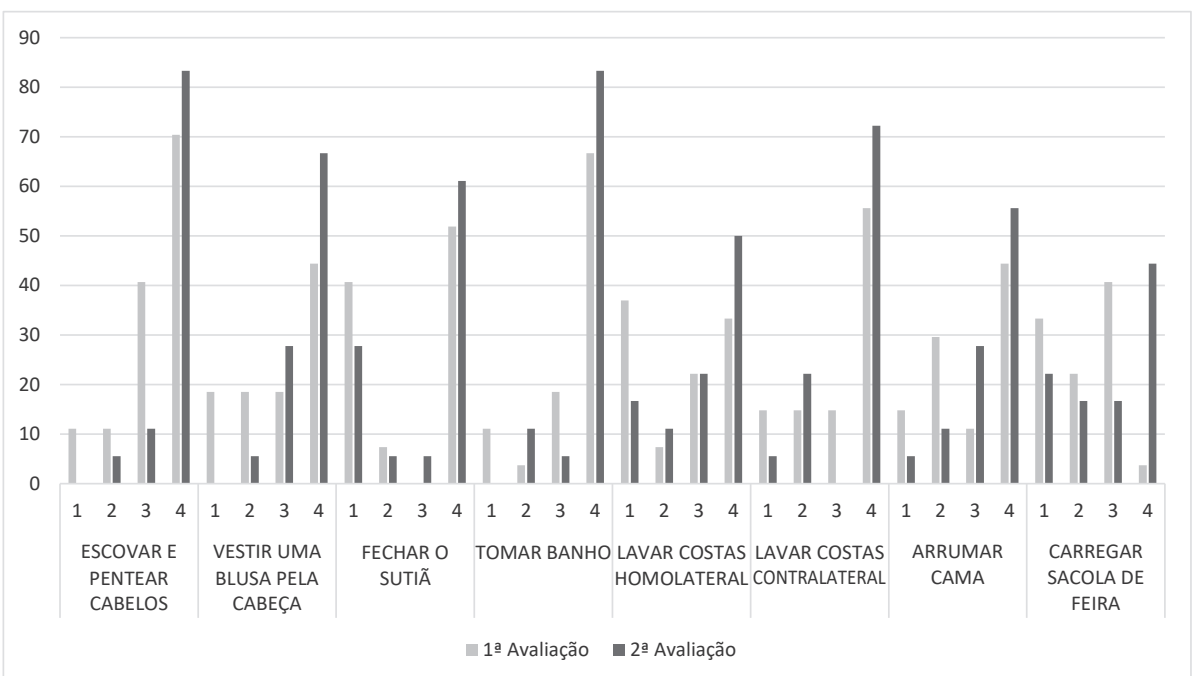

Figura 2. Frequência em porcentagem de respostas das voluntárias na avaliação funcional proposta pela ficha de anamnese 
quanto maior o escore, melhor a capacidade funcional das

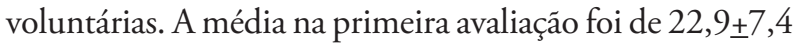
e, na reavaliação, foi de $26,7 \pm 6,1$, com $p=0,047$.

O escore do DASH apresentou valor de $38,61 \pm 26,9$ na avaliação e $32,15 \pm 25,27$ após quatro meses ( $\mathrm{p}=0,048$ ).

Ao considerar o tipo de cirurgia, os escores mais altos em ambas as avaliaçôes são notáveis naquelas mulheres submetidas a cirurgias radicais, o escore na primeira avaliação foi de $52,14 \pm 27,2$ e, após quatro meses, de $45,28 \pm 34,3$ ( $\mathrm{p}=0,016)$; enquanto, naquelas que realizaram cirurgia conservadora, os escores foram de $34,5 \pm 26,1$ na primeira avaliaçấo e de 29,7 $\pm 23,09$ ( $\mathrm{p}=0,176)$, ementando que escore alto indica maior disfunção.

Além disso, foram calculadas as médias das perguntas respondidas, quanto menor a média, melhor a capacidade funcional. O cálculo das médias foi efetuado somando as multiplicaçóes dos valores para cada nível da questáo pelo número de voluntárias que responderam tal nível dividido pelo número total de voluntárias (média ponderada). Com isso, as atividades propostas que reduziram mais a média entre as avaliaçóes foram "preparar uma refeição", indo de 2,40 na avaliação para 1,58 na reavaliação; "colocar um objeto em uma prateleira acima da cabeça" de 2,90 para 1,53; "lavar as costas" de 2,73 para 2,32; e "usar uma faca para cortar alimentos" de 2,43 para 2,05.

A maioria das questóes do questionário DASH reduziu a média dos valores de respostas na reavaliação. Apenas cinco questôes obtiveram médias maiores na reavaliaçáo, sendo elas: "abrir um frasco novo com uma tampa bem fechada" (de 3,07 para 3,37), "abrir ou empurrar uma porta pesada" (de 2,33 para 2,42), "realizar tarefas domésticas pesadas" (de 3,20 para 3,63), "fazer jardinagem ou trabalhar no quintal" (de 2,60 para 2,84) e "dormência no braço ou mão" (de 2,47 para 2,79).

As correlações entre o questionário DASH e a avaliação funcional foram $r=-0,82(p=0,02)$, com a idade $r=-0,574$ $(\mathrm{p}=0,783)$ e com o tempo entre cirurgia e avaliação $\mathrm{r}=0,558(\mathrm{p}=0,37)$. As correlaçóes entre o DASH com os movimentos de flexão e extensão de ombros homolaterais à cirurgia foram $\mathrm{r}=-0,52(\mathrm{p}=0,48)$ e $\mathrm{r}=-0,706(\mathrm{p}=0,782)$, respectivamente, e, entre a avaliação funcional do protocolo e a flexão e extensão do ombro homolateral, foram de $r=0,659(p=0,581)$ e $r=0,641 \quad(p=0,842)$, respectivamente.

\section{DISCUSSÃO}

Este estudo contou com a participação de mulheres diagnosticadas com câncer de mama, operadas e em acompanhamento em uma instituição pública de referência regional, onde o tratamento fisioterapêutico se deu em fase ambulatorial, e teve como objetivo analisar as características dos movimentos de ombro e cotovelo de mulheres submetidas à cirurgia oncológica mamária e relacioná-las com o ganho funcional conquistado por quatro meses de intervenção fisioterapêutica individualizada com eletroterapia e cinesioterapia, realizadas de duas a três vezes por semana, levando em conta os tipos cirúrgicos e as variáveis independentes presentes no contexto do estudo.

$\mathrm{Na}$ primeira avaliação, a análise da $\mathrm{ADM}$ pela biofotometria demonstrou que as mulheres apresentam diminuição dos movimentos no membro homolateral, independentemente do tipo de cirurgia. Resultado semelhante foi descrito quando analisaram-se 148 mulheres mastectomizadas, descrevendo perda significativa na $\mathrm{ADM}$, avaliada pela biofotometria, com diferença entre membros de até $20^{\circ}$ na flexão de ombro, sendo o movimento com maior prevalência de acometimento ${ }^{26}$, afirmando que a biofotometria é um instrumento útil para a mensuração da ADM nesse tipo de paciente.

Os resultados mostraram que os movimentos de abdução e flexão de ombro dos membros contralaterais à cirurgia foram significativamente maiores em relação aos movimentos do membro homolateral, corroborando o fato de que a intervenção cirúrgica no câncer de mama altera a ADM desses movimentos. Esses resultados foram semelhantes aos trabalhos, cujo objetivo também incluiu a mensuração da ADM de MMSS de mastectomizadas ${ }^{27,06}$. A restrição dos movimentos provavelmente se dá pela presença de fibroses decorrentes da radioterapia, bem como aderências cicatriciais, e quadros álgicos, enfatizados em mastectomias radicais ${ }^{28}$, que geram maior comprometimento.

$\mathrm{Na}$ amostra estudada, houve prevalência das cirurgias conservadora $(76,6 \%)$ e radical $(23,3 \%)$, semelhante com a síntese de dados do INCA em 2019, e com os resultados apresentados por Souza et al. ${ }^{29}$, que afirmam que esse tipo cirúrgico tem aumentado ao longo dos anos.

A redução funcional em pacientes mastectomizadas é descrita em diversos estudos ${ }^{19,31}$ realizados por meio de questionários. Em um estudo com 105 mulheres, os autores descreveram que a capacidade funcional está reduzida, com maior comprometimento das atividades que necessitam da abdução e/ou flexão de ombro, entretanto, com o tratamento fisioterapêutico, a ADM tem retornado ao normal na maioria dos casos e aumentado a funcionalidade ${ }^{30}$. Outro estudo descreve que as vivências emocionais também podem reduzir a funcionalidade dessas mulheres ${ }^{19}$. Nesse estudo, todas as participantes tinham acompanhamento psicológico concomitante ao tratamento fisioterapêutico, minimizando tal interferência.

O questionário DASH permite a avaliação adequada da capacidade funcional de MMSS em pacientes 
mastectomizadas ${ }^{28,31-33}$. A diferença de evolução de respostas no questionário foi proporcional em relação ao tipo de cirurgia, mesmo que tenha ocorrido redução maior em seu escore naquelas submetidas à cirurgia radical. Entretanto, houve uma baixa relação entre a redução dos movimentos de abdução e flexão de ombro, independentemente do tipo cirúrgico, entre as variáveis presentes no estudo. Com isso, pode-se dizer que a capacidade funcional de MMSS, embora muito impactada pela $\mathrm{ADM}$, é dependente de mais fatores do contexto biopsicossocial da voluntária, como fatores ambientais e pessoais ${ }^{19}$.

A literatura apresenta vários recursos fisioterapêuticos com evidência para o período pós-cirúrgico por Pereira et al. ${ }^{34}$; Petito et al..$^{35}$; Kneis ${ }^{36}$; e Oliveira et al. ${ }^{37}$. Trata-se de protocolos de atendimentos que objetivam a melhora da qualidade de vida (23\%), a melhora da funcionalidade e independência $(13,5 \%)$ e açôes preventivas de complicaçóes $(9,5 \%)$, o que não comprovam uma delimitaçáo clara para os objetivos da intervençáo, sendo que o primordial para esses pacientes deveria ser o retorno à funcionalidade e, consequentemente, a melhora da qualidade de vida, independente da intervenção ${ }^{38}$. O trabalho aqui apresentado mostra que houve ganho funcional significativo, o que certamente irá impactar na melhora da qualidade de vida com a intervenção executada, porém cabe a outro estudo analisar quais intervençóes são mais eficazes para atingir tal objetivo.

O estudo apresentou limitaçôes quanto ao número amostral para realização das análises correlacionais, justificado pela rotina do setor de fisioterapia da instituição suprir toda a demanda; para tanto, tais dados podem náo representar o cenário real de mulheres com a condição de saúde do câncer de mama. Entretanto, em razáo do embasamento e do confrontamento favorável com a literatura aqui apresentada, este estudo favorece a realização de mais pesquisas que envolvam análises da ADM nos diferentes tipos cirúrgicos e seus impactos na capacidade funcional, além de promover informaçóes acerca da realidade dos serviços públicos de saúde sobre o câncer de mama.

\section{CONCLUSÃO}

Observou-se, neste estudo, que as voluntárias apresentaram limitação nos movimentos de abdução e flexão de ombro homolateral quando comparado ao contralateral e, após intervenção fisioterapêutica individualizada com eletroterapia e cinesioterapia, tais movimentos aumentaram proporcionalmente em ambos os tipos cirúrgicos, em maior grau naquelas submetidas à cirurgia radical, a qual também causou maior impacto nesses movimentos. Além disso, houve melhora no ganho funcional avaliado pelos questionários.

Portanto, verificou-se que o ganho na ADM de abduçáo e flexão de ombro nessas pacientes acarreta ganho funcional em ambos os tipos cirúrgicos.

\section{CONTRIBUIÇÕES}

Todos os autores contribuíram substancialmente na concepção e/ou no planejamento do estudo; na obtenção, na análise e/ou interpretação dos dados; assim como na redação e/ou revisão crítica e aprovaram a versão final a ser publicada.

\section{DECLARAÇÃO DE CONFLITO DE INTERESSES}

Nada a declarar.

\section{FONTES DE FINANCIAMENTO}

Conselho Nacional de Desenvolvimento Científico e Tecnológico (CNPq).

\section{REFERÊNCIAS}

1. Instituto Nacional de Câncer José Alencar Gomes da Silva. Estimativa 2020: incidência de câncer no Brasil [Internet]. Rio de Janeiro: INCA; 2020 [acesso 2020 jun 18]. Disponível em: https://www.inca.gov. br/publicacoes/livros/estimativa-2020-incidencia-decancer-no-brasil

2. Freitas Júnior R, Nunes RD, Martins E, et al. Fatores prognósticos do câncer de mama e sobrevida global em cinco e dez anos na cidade de Goiânia, Brasil: estudo de base populacional. Rev Col Bras Cir. 2017;44(5):435-443. doi: https://doi.org/10.1590/0100-69912017005003

3. Martins CA, Guimaraes RM, Silva RLPD, et al. Evolução da mortalidade por câncer de mama em mulheres jovens: desafios para uma política de atenção oncológica. Rev Bras Cancerol. 2013;59(3):341-349.

4. Santos DB, Vieira EM. Imagem corporal de mulheres com câncer de mama: uma revisão sistemática da literatura. Rev Ciên Saúde Col. 2011;16(5):2511-22. doi: http://dx.doi.org/10.1590/S1413-81232011000500021

5. Jammal MP, Machado ARM, Rodrigues LR. Fisioterapia na reabilitação de mulheres operadas por câncer de mama. Mundo Saúde. 2008;32(4):506-510.

6. Bregagnol RK, Dias AS. Alteraçóes funcionais em mulheres submetidas à cirurgia de mama com linfadenectomia axilar total. Rev Bras Cancerol. 2010;56(1):25-33.

7. Santos RO, Luz KRG. Complex decongestive physiotherapy in lymphedema post-mastectomy. ReonFacema. 2016;2(1):177-80. 
8. Casassola GM, Gonçalves GR, Stallbaum JH, et al. Intervençôes fisioterapêuticas utilizadas na reabilitação funcional do membro superior de mulheres pósmastectomia. Fisio Brasil. 2020;21(1):93-103. doi: http://dx.doi.org/10.33233/fb.v21i1.2786

9. Gouveia VHO, Araújo AGF, Maciel SS, et al. Confiabilidade das medidas inter e intra-avaliadores com goniômetro universal e flexímetro. Fisioter Pesq. 2014;21(3):229-235. doi: https://doi.org/10.590/18092950/52921032014

10. Amadio AC. Modelagem do controle postural humano. In: IX Congresso Brasileiro de Ciências do Esporte, 2001 maio 29 a jun 1; Gramado, RS.

11. Baraúna MA, Canto RST, Schulz E, et al. Avaliação da amplitude de movimento do ombro em mulheres mastectomizadas pela biofotogrametria computadorizada. Rev Bras Cancerol. 2004;50(1):27-31.

12. Barbosa JAN, Amorim MHC, Zandonade E, et al. Avaliação da postura corporal em mulheres com câncer de mama. Rev Bras Ginecol Obstet. 2013;35(5):215-20. doi: https://doi.org/10.1590/S0100-72032013000500005

13. Bergmann A, Mattos IE, Koifman RJ, et al. Morbidade após o tratamento para câncer de mama. Fisio Brasil. 2000;1(2):101-8. doi: http://dx.doi.org/10.33233/ fb.v1i2.619

14. Rietman JS, Dijkstra PU, Geertzen JHB, et al. Shortterm morbidity of the upper limb after sentinel lymph node biopsy or axillary lymph node dissection for stage I or II breast carcinoma. Cancer. 2003;98(4):690-6. doi: https://doi.org/10.1002/cncr.11545

15. Hayes SC, Rye S, Battistutta D, et al. Upper-body morbidity following breast cancer treatment is common, may persist longer-term and adversely influences quality of life. Health Qual Life Outcomes. 2010;8:92. doi: https://doi.org/10.1186/1477-7525-8-92

16. Smoot B, Wong J, Cooper B, et al. Upper extremity impairments in women with or without lymphedema following breast cancer treatment. J Cancer Surviv. 2010;4(2):167-78. doi: https://doi.org/10.1007/s11764010-0118-x

17. Orfale AG. Tradução e validação do disabilities of the arm, shoulder and hand (DASH) para a língua portuguesa [dissertação]. São Paulo: Escola Paulista de Medicina, Universidade Federal de São Paulo (UNIFESP); 2003.

18. Assis MR. Comprometimento funcional tardio do membro superior e qualidade de vida de mulheres submetidas a cirurgia do câncer de mama [dissertação]. São Carlos (SP): Universidade de São Carlos; 2012.

19. Nicolussi AC, Sawada NO. Qualidade de vida de pacientes com câncer de mama em terapia adjuvante. Rev Gaúch Enferm. 2011;32(4):759-66. doi: https:// doi.org/10.1590/S1983-14472011000400017

20. Ferreira da Silva RL, Coelho RR, Barreto GA, et al. Comparação entre a avaliação da amplitude articular estática do cotovelo por meio de três diferentes métodos: goniometria, biofotogrametria e goniometria da imagem radiológica. Fisio Brasil. 2009;10(2):106-112. doi: http:// dx.doi.org/10.33233/fb.v10i2.1511

21. Nascimento FC, Flausino TC. Biofotogrametria: a utilização do software de avaliação postural (SAPO). Rev Eletr Saúde Ciên. 2015;5(1):36-51.

22. Watson AW, MacDonncha C. A Reliable technique for the assessment of posture: assessment criteria for aspects of posture. J Sports Med Phys Fitness, 2000;40(3):260-270.

23. Iunes DH. Análise da confiabilidade inter e intraexaminador na avaliação postural pela fotogrametria computadorizada [dissertação]. Ribeirão Preto (SP): Faculdade de Medicina de Ribeirão Preto; 2005. doi: http://dx.doi.org/10.11606/D.17.2005.tde-30042008153042

24. Drumond AS. Exploração do disabilities arm, shoulder and hand (DASH) através da classificação internacional de funcionalidade, incapacidade e saúde (CIF) e da análise rasch [dissertação]. Belo Horizonte (MG): Universidade Federal de Minas Gerais; 2006.

25. Weber JC, Lamb DR. Statistics and research in physical education. St Luis: Mosby Comp; 1970.

26. Young AE. The surgical management of early breast cancer. Int J Clin Pract. 2001;55(9):603-8.

27. Nascimento SL, Oliveira RR, Oliveira MMF, et al. Complicaçôes e condutas fisioterapêuticas após cirurgia por câncer de mama: estudo retrospectivo. Fisioter Pesqui. 2012;19(3):248-255. doi: http://dx.doi. org/10.1590/S1809-29502012000300010

28. Bergmann A, Mattos IE, Koifman RJ. Fatores de risco para linfedema após câncer de mama: uma revisão da literatura. Fisioter Pesq. 2008;15(2):207-13. doi: https:// doi.org/10.1590/S1809-29502008000200016

29. Souza E, Carvalho FN, Bergmann A, et al. Funcionalidade de membro superior em mulheres submetidas ao tratamento do câncer de mama. Rev Bras Cancerol. 2013;59(3):409-417.

30. Britto JS, Marcelino JFQ. Desempenho ocupacional de mulheres submetidas à mastectomia. Cad Ter Ocup UFSCar. 2014;22(3):473-85. doi: https://doi. org/10.4322/cto.2014.068

31. Rett MT, Santos AKG, Mendonça ACR, et al. Efeito da fisioterapia no desempenho funcional do membro superior no pós-operatório de câncer de mama. Rev Ciênc Saúde. 2013;6(1) 18-24. doi: http://dx.doi. org/10.15448/1983-652X.2013.1.11375

32. Cerdeira DQ, Nunes TTV, Lima AC, et al. Atuação fisioterapêutica em pacientes pós-cirurgia do câncer de mama: uma revisão bibliográfica. Rev Expr Católica. 2014;3(1):23-35.

33. Recchia TL, Prim AC, Luz CM. Upper limb functionality and quality of life in women with fiveyear survival after breast cancer surgery. Rev Bras 
Ginecol Obstet. 2017;39(3):115-22. doi: https://doi. org/10.1055/s-0037-1598642

34. Pereira CMA, Vieira EORY, Alcântara PSM. Avaliação de protocolo de fisioterapia aplicado a pacientes mastectomizadas a Madden. Rev Bras Cancerol. 2005;51(2):143-148.

35. Petito EL, Nazário ACP, Martinelli SE, et al. Aplicação de programa de exercícios domiciliares na reabilitação do ombro pós-cirurgia por câncer de mama. Rev LatinoAm Enfermagem. 2012;20(1):35-43. doi: https://doi. org/10.1590/S0104-11692012000100006

36. Kneis S, Wehrle A, Ilaender A, et al. Results from a pilot study of handheld vibration: exercise intervention reduces upper-limb dysfunction and fatigue in breast cancer patients undergoing radiotherapy: VibBRa study. Integr Cancer Ther. 2018;17(3):717-27. doi: https://doi. org/10.1177/1534735418766615

37. Oliveira MMF, Souza GA, Miranda MS, et al. Upper limbs exercises during radiotherapy for breast cancer and quality of life. Rev Bras Ginecol Obstet. 2010;32(3):133-8. doi: https://doi.org/10.1590/S010072032010000300006

38. Borges CAM, Silveira CF, Lacerda PCMT, et al. Análise dos métodos de avaliação, dos recursos e do reconhecimento da fisioterapia oncológica nos hospitais públicos do Distrito Federal. Rev Bras Cancerol. 2008;54(4):333-44. 